
\title{
25 Research Soure \\ Potential Contribution of the Uterine Microbiome in Early Missed Abortion
}

\section{junjun shu}

Nanchang University Medical College: Medical College of Nanchang University https://orcid.org/00000002-9742-1332

\section{ShiXin Lin}

Nanchang University Medical College: Medical College of Nanchang University https://orcid.org/00000001-5389-0332

\section{Yu Wu}

Nanchang University Medical College: Medical College of Nanchang University

\section{Dan Gong}

Nanchang University Medical College: Medical College of Nanchang University

\section{Xia Zou}

Nanchang University Medical College: Medical College of Nanchang University Hong Zhu

Nanchang University Medical College: Medical College of Nanchang University Jun Gao ( $\nabla$ drgaojun@yeah.net )

Nanchang University Medical College: Medical College of Nanchang University https://orcid.org/00000003-4086-1086

\section{Research Article}

Keywords: missed abortion, healthy pregnant, microbiome, bioinformatic analysis, 16s-rRNA sequencing, Uterine microbiome, female reproductive tract

Posted Date: July 26th, 2021

DOI: https://doi.org/10.21203/rs.3.rs-623634/v1

License: (9) This work is licensed under a Creative Commons Attribution 4.0 International License. Read Full License 


\section{Abstract}

\section{Background}

Missed abortion is a kind of pregnancy failure caused by various reasons. The etiology is complicated, and the incidence of miscarriage is increasing in recent years. Previous studies shown microbiota contributes to multi-systemic function, whereas the relationship between microbiota and early missed abortion remains unknown. This study aimed to explore the composition of uterine microbiota in missed abortion and the potential role.

Methods

We enrolled 19 patients diagnosed with missed abortion and 12 healthy pregnant who subsequently had 6-8th week pregnant. All samples were taken from the endometrial fluid by a special disposable endometrial sampler. After samples were collected, DNA was extracted and amplified. The highthroughput next-generation sequencing (MiSeq) of the 16S rDNA V3-V4 region was used to identify the present of microbiota. The a-diversity of microbiota data was used to reflect species richness and evenness within bacterial populations, $\beta$-diversity was used to reflect the shared diversity between bacterial populations, and Nonmetric Multidimensional Scaling based on Weighted Unifrac distance. Statistical was determined by use of multiple testing, including the generalized mixed-effects model.

Results

The microbiome sequencing (16S rDNA V3-V4 region) revealed that low abundance microbiome was detected in uterine cavity of patients with missed abortion and normal pregnancy. The diversity of intrauterine microflora in patients with missed abortion was higher than that in patients with induced abortion in normal pregnancy. There was no significant difference in alpha diversity between the two groups, but a significant difference was observed in beta diversity. PCoA moment array analysis did not show significant differences. Proteobacteria and Firmicutes in missed abortion patients were significantly more than those in normal pregnancy group.

\section{Conclusion}

There are low abundance of microflora in uterine cavity of missed abortion patients, and the diversity of microflora is higher than that of normal pregnancy patients. Also, Proteobacteria and Firmicutes may be potential biological markers in missed abortion. New observations may prompt further investigations to understand the potential mechanism of microbiology on pathologic human pregnancy in the future.

Subjects

Microbiology, Molecular Biology, Gynecology and Obstetrics, missed abortion, Female Reproductive Tract,

\section{Introduction}


Missed abortion[1] refers to the embryo or fetus has stranded intrauterine, which means that the embryo or fetus has died and failed to discharge in time. Due to its processing difficulties, it often causes serious damage to the endometrium, leading to endometrial fibrosis, resulting in intrauterine adhesions, and even female secondary infertility which serious impact on women's reproductive health[2]. In recent years, with the development of society, followed by environmental pollution, female physiological and psychological changes, the incidence of missed abortion is climbing year by year[3]. Research on its etiology has always been a hot topic. Many studies have shown that missed abortion is related to chromosomal abnormalities and changes in maternal hormone levels, etc. However, recently, researchers have gradually paid attention to whether the microecological environment of uterine cavity and uterine implantation is suitable for the growth of embryonic tissue.

The female upper reproductive tract, which includes the uterus and fallopian tubes, has long been considered sterile[4,5]. New studies have emerged in an endless stream in the recent decades. It has been reported that after bacterial culture analysis of endometrial samples, uterine is actually a bacteria-bearing environment with a complex uterine microecological environment system, which challenges the view that uterine cavity is unsterile[6].Now, a new research method, 16S rRNA sequencing[7], has been contributed to the study of intrauterine microenvironment (this method excludes the interference of bacterial culture), which also proves the possibility of bacterial colonization in the uterus[8].

Previous studies have shown that endometriosis[9], endometrial cancer[10], and preeclampsia[11] are associated with the microflora of the female upper reproductive tract, nevertheless there still are few studies on the microflora in uterine cavity of patients with missed abortion.

In this study, based on the high-throughput sequencing of V3 and V4 of the high variable region of $16 \mathrm{~S}$ ribosomal RNA (rRNA) gene, the intrauterine microflora of patients with missed abortion and normal pregnancy undergoing induced abortion was analyzed. We aim to compare the characteristics and diversity of microecological flora in uterine cavity between early missed abortion and healthy pregnancy from a multi-dimensional perspective. Further to explore the important categories of bacteria with significant differences, dig deep into biological markers, study the influence of uterine microecological environment on missed abortion from different perspectives, and find out the functional flora, so as to provide theoretical basis for the subsequent development of functional drugs.

\section{Methods}

\section{Patient recruitment and ethical considerations}

Between Jan to March 2021, patients treated in the first hospital of Nanchang were recruitment. Compare the differences in the intrauterine microecological environment between 6-8 weeks of normal pregnancy and missed abortion. All research participants gave their written informed consent for uterine liquid sample collection and subsequent microbiological analysis. All experiments steps were performed followed by the declaration of Helsinki (as was revised in 2013). This study was approved by the Medical Ethical Committee of The First Hospital of Nanchang (ID 2020047), Nanchang, China. 


\section{Patient characteristics}

29 Patients enrolled in this study. Among them: 12 cases because of the undesired pregnancy requirements of induced abortion women, 17 cases of missed abortion directly by negative pressure suction to terminate pregnancy. The inclusion criteria included the items: patients within 18-36 years old, with regular menstrual cycle ( $28 \pm 5$ days), without antibiotics using in past 1 month, no hormone replacement therapy in past 1 month, no genitourinary tract infection or infection in other parts of the body within 3 months, no douching, vaginal medications and sexual activity within 72 hours. The exclusion criteria included the following items: patients with pelvic inflammatory disease, cervical inflammation, bacterial vaginosis and any other acute inflammation; patients with diabetes, cancer and autoimmune disorders.

\section{Sample collection}

All samples were collected on the day of vacuum aspiration surgical abortion. In order to minimize the contamination, the cervical canal and vaginal was sterilized with iodine before sampling. All procedures were performed by two senior gynecologists ( $Y u$ W and $D a n ~ G)$ with experience in performing intra-uterine procedures and following a strict study protocol. Disposable endometrial sampler (Jiangsu Jiadingcheng Medical Instrument Co., Lt) was used for sampling the endometrium. The special device, which is similar to the Tao brush[12], has a plastic protective cover on the outside of the brush to ensure that the brush will not be contaminated as it passes through the vagina and cervix. It was inserted directly into the uterine cavity to avoid any contact with the vaginal wall. Then, the samples were stored in a sterile Falcon at $-80^{\circ} \mathrm{C}$ until DNA extraction was performed and further analysis. In this study, when used correctly, the brush can minimize the risk of cervicovaginal contamination and ensure the result.

\section{DNA extraction, bacterial 16S rRNA amplification and high-throughput sequencing}

The total genomic DNA of the samples was extracted by SDS method, and then the purity and concentration of the DNA were detected by agarose gel electrophoresis. An appropriate amount of the sample DNA was put into a centrifuge tube, and the sample was diluted to $1 \mathrm{ng} / \mu \mathrm{L}$ with sterile water. After that, the 16s-rRNA genes of distinct regions (16SV3-V4) were amplified using specific primers $338 \mathrm{~F}\left(5^{\prime}-\right.$ ACTCCTACGGGAGGCAGCA-3'\and 806R (5'-GGACTACHVGGGTWTCTAAT-3') with the barcode. All PCR reactions were carried out with $15 \mu \mathrm{L}$ of Phusion ${ }^{\circledR}$ High-Fidelity PCR Master Mix (New England Biolabs) to ensure the amplification efficiency and accuracy. Mix same volume of $1 \mathrm{X}$ loading buffer (contained SYB green) with PCR products and operate electrophoresis on $2 \%$ agarose gel for detection. PCR products was mixed in equidensity ratios. Then, mixture PCR products was purified with Qiagen Gel Extraction Kit (Qiagen, Germany). Sequencing libraries were generated using TruSeq ${ }^{\circledR}$ DNA PCR-Free Sample Preparation Kit (Illumina, USA) following manufacturer's recommendations and index codes were added. The library quality was assessed on the Qubit@ 2.0 Fluorometer (Thermo Scientific) and Agilent Bioanalyzer 2100 system. Then, the library was sequenced on an Illumina NovaSeq platform and 250 bp paired-end reads were generated. 


\section{Bioinformatic Analysis}

Sequences analysis were performed by Uparse software (Uparse v7.0.1001 http://drive5.com/uparse/ ) [13]. Sequences with $\geq 97 \%$ similarity were assigned to the same OTUs. Representative sequence for each OTU was screened for further annotation. For each representative sequence, the Silva Database (http://www.arb-silva.de/)[14] was used based on Mothur algorithm to annotate taxonomic information. In order to study phylogenetic relationship of different OTUs, and the difference of the dominant species in different samples(groups), multiple sequence alignment were conducted using the MUSCLE software (V ersion 3.8.31 http://www.drive5.com/muscle/)[15]. Alpha diversity[16] is applied in analyzing complexity of species diversity for a sample through 6 indices, including Observed-species, Chao1, Shannon, Simpson, ACE, Good-coverage. All these indices in our samples were calculated with QIIME (V ersion 1.7.0) and displayed with $R$ software (V ersion 2.15.3). Beta diversity $[17,18]$ analysis was used to evaluate differences of samples in species complexity, Beta diversity on both weighted and unweighted unifrac were calculated by QIIME software (V ersion 1.9.1). Cluster analysis was preceded by principal component analysis (PCA), which was applied to reduce the dimension of the original variables using the FactoMineR package and ggplot2 package in $\mathrm{R}$ software (V ersion 2.15.3). Principal Coordinate Analysis ( $P C \circ A)$ was performed to get principal coordinates and visualize from complex, multidimensional data. A

distance matrix of weighted or unweighted unifrac among samples obtained before was transformed to a new set of orthogonal axes, by which the maximum variation factor is demonstrated by first principal coordinate, and the second maximum one by the second principal coordinate, and so on. PCoA analysis was displayed by WGCNA package, stat packages and ggplot2 package in $\mathrm{R}$ software ( $\mathrm{V}$ ersion 2.15.3).

\section{Statistical analysis}

Statistical analysis was performed with SPSS 22.0 software (version 22, IBM). The differences between two groups were analyzed by Student's t-test or Mann-Whiter $U$ test for quantitative data. Categorical variables were expressed as numbers (\%) and performed by the chi-squared test or Fisher's exact. The statistical significance was considered at two-side $\mathrm{P}<0.05$.

\section{Results}

\section{Samples and participant characteristics}

A total of 29 patients enrolled in this study. Of these, 12 women were normal pregnant with $6-8 w$ which we called the "ab" group, 17 women were diagnosed with missed abortion at 6-8w which we named "pf" group. The average age of the patients was 27.2 (range 18.0-36.0). The average gestational age was 6.8 weeks (range 6.0-8.0). There was no difference between each sample in age, BMl, and pregnant time $(p>0.05)$.

\section{Microbiome characterization}


Based on Illumina Nova sequencing platform, PCR free library was constructed, and then double end sequencing was performed. Through the splicing of reads, 96649 tags were measured on average for each sample, and 85759 valid data were obtained after quality control. The quantity of effective data under quality control reached 62,048 , and the effective rate of quality control was $64.72 \%$. The quantity of quality control valid data was 62048 , and the effective rate of quality control was $64.72 \%$. The sequences were clustered into OTUs (operational taxonomic units) with $97 \%$ identity, and a total of 2124 OTUs were obtained. The OTUs sequences were annotated with silva138 database. A total of 637 (29.99\%) OTUs were annotated to genus level.

According to the results of species annotation, the species with the abundance content of top5 at each level are selected to display the Sangji map, from which the distribution trend of individual species in different samples can be seen. Taking the gate level results as an example, the Sangji map results are as follows (figure 1). From the test results of all samples, the top five species were Proteobacteria, Firmicutes, Actinobacteriota, Bacteroidota, Acidobacteriota. Between the two groups, Firmicutes, Actinobacteriota, Bacteroidota, and Acidobacteriota in the pregnant failed group were all more than those in the healthy pregnancy group, while Proteobacteria were less than those in the normal pregnancy group.

After the OTUs are obtained, the rarefaction curve is drawn to judge whether the current sequencing depth of each sample is enough to reflect the microbial diversity of the community sample. Sparse curve is a common method in the field of ecology, through a random sample from each sample a certain number of sequence (that is, the amounts of samples in no more than the existing sequencing of a certain depth under deep sampling), the sample can be predicted in a given range of sequencing depth, that may contain in the total number of species and the relative abundance of each species. Therefore, under the same sequencing depth, the number of OTU in different samples is compared, so as to measure the diversity of each sample to some extent (figure 2).

\section{Species annotation}

By comparing with the database SILVA138, species annotation was carried out, and statistics of different taxonomic levels showed that: There were 2,124 OTUs in total, among which 1,780(83.80\%) OTUs could be annotated to the database, $83.80 \%$ could be annotated to the kingdom level, $49.29 \%$ could be annotated to the phylum level, $48.45 \%$ could be annotated to the class level, $47.27 \%$ could be annotated to the order level, and $42.00 \%$ could be annotated to the family level, The proportion of genus level was $29.99 \%$, and the proportion of species level was $8.95 \%$.(table 1 )

\section{Table 1 Species annotation}




\begin{tabular}{|ll|}
\hline OTU catalogue & 2,124 \\
\hline Annotated on database & $1,780(83.80 \%)$ \\
\hline Annotated on Unclassified & $344(16.20 \%)$ \\
\hline Annotated on Kingdom level & $83.80 \%$ \\
\hline Annotated on Phylum level & $49.29 \%$ \\
\hline Annotated on Class level & $48.25 \%$ \\
\hline Annotated on Order level & $47.27 \%$ \\
\hline Annotated on Family level & $42.00 \%$ \\
\hline Annotated on Genus level & $29.99 \%$ \\
\hline Annotated on Species level & $8.95 \%$ \\
\hline
\end{tabular}

At the kingdom level, we found that Proteobacteria, Firmicutes and Actinobacteriota were the dominant ones. At class level, the dominant bacteria were Gammaproteobacteria, Bacilli, and Actinobacteria. The dominant species at order level were Burkholderiales, Lactobacillales and Bifidobacteriales. At the family level, the dominant species were Oxalobacteraceae, Lactobacillaceae and Bifidobacteriaceae. At the genus level, the dominant species were Herbaspirillum, Lactobacillus and Gardnerella. The dominant species were Herbaspirillum_huttiense, Lactobacillus_iners, Stenotrophomonas_nitritireducens. In order to further study the phylogenetic relationships of species at the genus level, the representative sequences of TOP100 genera were obtained through multiple sequence alignment and shown in Fig 3

\section{Alpha Diversity}

The figure below shows Beeswarm (figure 4). The figure on the left (figure 4a) shows the scatter distribution of the total number of species among different groups of all samples, namely, Richness; the figure on the right (figure $3 \mathrm{~b}$ ) is the comparison of Shannon's diversity index, which reflects the differences in diversity and evenness among different samples. As shown in the figure below, through Wilcoxon rank sum test, it was found that the number of species measured was significantly different between $A B \_P F$ groups, with a significant $P$ value of 0.0053 . There was no significant difference in Shannon index among AB_PF groups, with a significant $P$ value of 0.0973 .

\section{Beta Diversity}

Beta diversity is a comparative analysis of the microbial community composition of different samples. Unifrac distance (taking genetic sequence information between species as reference) and Bray-Curtis distance (considering species presence and abundance) are usually used for dimensional-reduction analysis. When the number of samples in each group is more than 5 , corresponding confidence circles can be added to the dimensional-reduction map. PCoA (principal co-coordinates analysis) is to extract the most important elements and structures from multi-dimensional data through a series of eigenvalues and 
eigenvectors. The analysis is generally based on the Unifrac distance, and the principal coordinate with the largest contribution rate is selected for drawing and display (figure 5). NMDS (non-metric multidimensional scaling) is a non-linear model, which can overcome the shortcomings of linear models (including PCA and PCOA) and better reflect the nonlinear structure of ecological data(figure 6).

\section{Species differences analysis between groups}

To identify species with significant differences between groups, T-tests, Metastat and Lefse were used to search for biomarkers with marker properties. From the perspective of species abundance at different levels, different species can be obtained by conventional T-test. The figure below (figure 7) show the average abundance of Firmicutes in $A B$ and PF is $9.7 \%$ and $29.1 \%$ respectively, and the significant $p$-value of this species between the two groups is found to be 0.0400 through testing.

Metastat method was used to perform hypothesis testing on species abundance data between groups to obtain $P$ values, which were corrected to obtain Q values. Finally, the species with significant differences were screened according to the $Q$ value, and the abundance distribution box chart of the different species among groups was drawn (figure 8). Proteobacteria in missed abortion group was significantly more than that in healthy pregnant. $(p<0.05)$

Lefse[19] analysis is used to detect the species diversity among different groups by rank sum test, and LDA (linear discriminant analysis) is used to reduce the dimension, so as to evaluate the impact of different species. The LDA score is obtained. Finally, the histogram of LDA value distribution of different species and the evolutionary branching diagram of different species are drawn. The figure (figure 9) below shown that through lefse analysis of PF group and AB group, we find that there are 13 biomarkers with LDA score > 4, including gamma Proteobacteria and Herbaspirillum, Huttiense, Herbaspirillum, oxalobacteraceae, burkholderiales, Firmicutes, etc.

\section{Network analysis}

The co-occurrence network map provides a new perspective for the study of community structure and function in complex microbial environment. Since the co-occurrence relationships of microorganisms in various environments are quite different, the influences of the environmental factors on the adaptability of microorganisms can be intuitively seen through the co-occurrence network diagram of species, as well as the dominant species and species groups interacting closely in a given environment. These dominant species and species groups often play a unique and important role in maintaining the stability of microbial community structure and function in the environment. In our study, after calculating the correlation index (Spearman correlation coefficient SCC or Pearson correlation coefficient PCC) for all samples, the filter conditions are set as follows: (1) the connection with correlation coefficient $₫ 0.6$ is removed, (2) the node self-connection is filtered out, (3) the connection with node abundance less than $\% 0.005$ is removed, and then the network diagram as shown in the following figure is obtained: (figure 10) 


\section{Discussion}

Missed abortion is a special type of spontaneous abortion which accompany serious complication endanger the reproductive healthy[20]. Previous studies on missed abortion focused on chromosome abnormalities[21], uterine artery blood flow resistance, angiogenesis[22], immune inflammationrelated[23, 24], etc. In recent years, with the further study of the human microbiome, researchers gradually realized that from the surface of human body to the intestinal flora, all of them have an impact on the ecological balance of human body and the occurrence and development of diseases. Human microbiology was correlation with obesity[25], colon cancer[26], and even Alzheimer's disease[27].

With the deepening of research, extended to the field of Obstetrics and Gynecology, intrauterine microenvironment is a rising topic in recent years. Previous scholars believe that because of the role of cervical mucus plug, the intrauterine cavity is sterile, even if the intrauterine device is inserted, it also produces aseptic inflammatory reaction, so as to play the role of contraception[28]. However, in recent years, due to the continuous progress of detection technology, even a small amount of microorganisms can be obtained by PCR amplification without the interference of bacterial culture. Therefore, we found that there are bacteria in the uterine cavity and even the whole upper genital tract. The healthy female upper reproductive tract is actually a state of bacteria containing a variety of microorganisms. All kinds of microorganisms coexist peacefully, forming a good micro ecological environment. Once this micro ecological environment is broken, it may lead to related diseases. It has been reported that intrauterine microbiome is associated with endometriosis[29] and endometrial cancer[30].

In view of this, our study focused on the intrauterine microenvironment of patients with missed abortion in early pregnancy. Our study found that there is a relatively low abundance of microbial flora in the uterine cavity of patients. For avoiding contamination of vaginal bacteria, we used a special sampler which was similar with Tao's brush[31] to sample endometrial microorganisms, thus minimizing the pollution. The composition of the uterine flora found in this study was significantly different from that of the known female vaginal flora [32,33], and the dominant bacteria were also different, indicating that the uterine flora found in this study was less likely to be from vaginal contamination. The top five species were Proteobacteria, Firmicutes, Actinobacteriota, Bacteroidota, Acidobacteriota. Among the microflora at the phylum level, previous studies have reported that Proteobacteria can be detected in endometriosis[34], uterine cavity with repeated implantation failure[8], and intrauterine growth restriction[35]. Our present study also detected it, suggesting that Proteobacteria plays a key role in the low-profile microbiome of the uterine cavity, but its specific mechanism needs to be further studied. On the other hand, compared with normal pregnancy, the number of Firmicutes in patients with early missed abortion increased significantly. It has previously been reported that Firmicutes predominate in patients with endometrial cancer[36]. It needs to be further explored whether it is related to immune inflammation in pregnant failed.

\section{Conclusion}


In conclusion, our study based on 16sRNA high-throughput sequencing, low abundance of microbiome was detected in the early pregnancy embryo termination group, and the diversity of microbiome in embryo termination group was higher than that in the normal pregnancy induced abortion group. The microflora diversity of pregnant failed was relatively high, which indicated that the increase of microflora diversity in uterine cavity was related to abnormal pregnancy. The results of our study also revealed that Proteobacteria and Firmicutes were the dominant microflora in patients with missed abortion, and there were differences between them and the uterine microflora of normal pregnancy. It may become a biomarker for microorganism in uterine cavity during embryo missed abortion. These findings may provide a novel view of the microbiome function-missed abortion cross-link system, in which the immune system is associated with the microbiota and missed abortion. However, further studies are also needed to understand such network. Furthermore, the present pilot study provides a novel concept that bacteria in upper genital tract may be correlated to the abnormal pregnant, especially with missed abortion. In future studies, we plan to establish the animal models which will also help to reveal the role of intrauterine flora composition in missed abortion and the possible mechanism. The bacterial features of uterine microorganism may become a non-invasive easy diagnostic method, and even bacterial therapy could be a possible method to help improve and treat microecological environment in uterine cavity with pregnant failed in near future.

\section{Abbreviations}

PCoA = principal co-coordinates analysis,

NMDS= non-metric multi-dimensional scaling

\section{Declarations}

Ethical Approval and Consent to participate: The research was approved by the Human Ethics Committee of The First Hospital of Nanchang (ID 2020047), Nanchang, China. Written informed consent was obtained from individual or guardian participants.

Consent for publication: Written informed consent for publication was obtained from all participants.

Availability of supporting data: All data generated or analyzed during this study are included in this published article.

Competing interests: The authors declare that they have no competing interests.

Funding: This study was supported in part by grants from the Science and Technology of Jiangxi Province, Jiangxi key R \& D plan (No. S2018ZPYFE0196)

\section{Authors' contributions}


Junjun Shu carried out the acquisition of data, analysis and interpretation of data and writing of the manuscript. ShiXin Lin has been involved in drafting the manuscript and revising it critically for important intellectual content. Yu Wu, Dan Gong collect the sample, Xia Zou do the analysis of data. Jun Gao and Hong Zhu conceived of the study, and participated in its design and coordination, helped to draft the manuscript and have given final approval of the version to be published. All authors have read and approved the final version of the manuscript.

\section{Competing interests}

The authors declare that they have no competing interests.

\section{Acknowledgements}

Funding from Dr Gao is gratefully acknowledged.

\section{References}

1. Wu, H.L., et al., Misoprostol for medical treatment of missed abortion: a systematic review and network meta-analysis. Sci Rep, 2017. 7(1): p. 1664.

2. Alves, C. and A. Rapp, Spontaneous Abortion, in StatPearls. 2021: Treasure Island (FL).

3. Fang, J., et al., Biochemical clinical factors associated with missed abortion independent of maternal age: A retrospective study of 795 cases with missed abortion and 694 cases with normal pregnancy. Medicine (Baltimore), 2018. 97(50): p. e13573.

4. Crha, I., et al., Uterine microbiome and endometrial receptivity. Ceska Gynekol, 2019. 84(1): p. 4954.

5. Koedooder, R., et al., Identification and evaluation of the microbiome in the female and male reproductive tracts. Hum Reprod Update, 2019. 25(3): p. 298-325.

6. Garcia-Grau, I., C. Simon, and I. Moreno, Uterine microbiome-low biomass and high expectationsdagger. Biol Reprod, 2019. 101(6): p. 1102-1114.

7. Watts, G.S., et al., $16 S$ rRNA gene sequencing on a benchtop sequencer: accuracy for identification of clinically important bacteria. J Appl Microbiol, 2017. 123(6): p. 1584-1596.

8. Kitaya, K., et al., Characterization of Microbiota in Endometrial Fluid and Vaginal Secretions in Infertile Women with Repeated Implantation Failure. Mediators Inflamm, 2019. 2019: p. 4893437.

9. Leonardi, M., et al., Endometriosis and the microbiome: a systematic review. BJOG, 2020. 127(2): p. 239-249. 
10. Laniewski, P., Z.E. Ilhan, and M.M. Herbst-Kralovetz, The microbiome and gynaecological cancer development, prevention and therapy. Nat Rev Urol, 2020. 17(4): p. 232-250.

11. Beckers, K.F. and J.L. Sones, Maternal microbiome and the hypertensive disorder of pregnancy, preeclampsia. Am J Physiol Heart Circ Physiol, 2020. 318(1): p. H1-H10.

12. Tao, L.C., Direct intrauterine sampling: the IUMC Endometrial Sampler. Diagn Cytopathol, 1997. 17(2): p. 153-9.

13. Edgar, R.C., UPARSE: highly accurate OTU sequences from microbial amplicon reads. Nat Methods, 2013. 10(10): p. 996-8.

14. Quast, C., et al., The SILVA ribosomal RNA gene database project: improved data processing and web-based tools. Nucleic Acids Res, 2013. 41(Database issue): p. D590-6.

15. Edgar, R.C., MUSCLE: multiple sequence alignment with high accuracy and high throughput. Nucleic Acids Res, 2004. 32(5): p. 1792-7.

16. Li, B., et al., Characterization of tetracycline resistant bacterial community in saline activated sludge using batch stress incubation with high-throughput sequencing analysis. Water Res, 2013. 47(13): p. 4207-16.

17. Lozupone, C. and R. Knight, UniFrac: a new phylogenetic method for comparing microbial communities. Appl Environ Microbiol, 2005. 71(12): p. 8228-35.

18. Lozupone, C., et al., UniFrac: an effective distance metric for microbial community comparison. ISME J, 2011. 5(2): p. 169-72.

19. Segata, N., et al., Metagenomic biomarker discovery and explanation. Genome Biol, 2011. 12(6): p. R60.

20. Vivas-Mejia, P., et al., c-Jun-NH2-kinase-1 inhibition leads to antitumor activity in ovarian cancer. Clin Cancer Res, 2010. 16(1): p. 184-94.

21. Zhao, L., H. Yang, and G. Liu, Relationship between age at menarche and chromosome numerical abnormalities in chorionic villus among missed abortions: A cross-sectional study of 459 women in China. J Obstet Gynaecol Res, 2020.

22. Zhi, Z., et al., Early missed abortion is associated with villous angiogenesis via the HIF1alpha/VEGF signaling pathway. Arch Gynecol Obstet, 2018. 298(3): p. 537-543.

23. Li, Y., et al., The Expression of PDCD4 in Patients With Missed Abortion and Its Clinical Significance. Reprod Sci, 2017. 24(11): p. 1512-1519. 
24. Luo, M., et al., The expression and clinical significance of three IncRNAs in patients with a missed abortion. Exp Ther Med, 2021. 21(1): p. 8.

25. Maruvada, P., et al., The Human Microbiome and Obesity: Moving beyond Associations. Cell Host Microbe, 2017. 22(5): p. 589-599.

26. Zeng, H., et al., Secondary Bile Acids and Short Chain Fatty Acids in the Colon: A Focus on Colonic Microbiome, Cell Proliferation, Inflammation, and Cancer. Int J Mol Sci, 2019. 20(5).

27. Sochocka, M., et al., The Gut Microbiome Alterations and Inflammation-Driven Pathogenesis of Alzheimer's Disease-a Critical Review. Mol Neurobiol, 2019. 56(3): p. 1841-1851.

28. Straub, T., M. Reynaud, and M. Yaron, [Intrauterine device and pelvic inflammatory disease: Myth or reality?]. Gynecol Obstet Fertil Senol, 2018. 46(4): p. 414-418.

29. Hernandes, C., et al., Microbiome Profile of Deep Endometriosis Patients: Comparison of Vaginal Fluid, Endometrium and Lesion. Diagnostics (Basel), 2020. 10(3).

30. Bracewell-Milnes, T., et al., Investigating the effect of an abnormal cervico-vaginal and endometrial microbiome on assisted reproductive technologies: A systematic review. Am J Reprod Immunol, 2018. 80(5): p. e13037.

31. Verstraelen, $\mathrm{H}$., et al., Characterisation of the human uterine microbiome in non-pregnant women through deep sequencing of the V1-2 region of the 16S rRNA gene. PeerJ, 2016. 4: p. e1602.

32. Chen, C., et al., The microbiota continuum along the female reproductive tract and its relation to uterine-related diseases. Nat Commun, 2017. 8(1): p. 875.

33. Witkin, S.S. and I.M. Linhares, Why do lactobacilli dominate the human vaginal microbiota? BJOG, 2017. 124(4): p. 606-611.

34. Akiyama, K., et al., Molecular detection of microbial colonization in cervical mucus of women with and without endometriosis. Am J Reprod Immunol, 2019. 82(2): p. e13147.

35. $\mathrm{Hu}, \mathrm{J} .$, et al., Intrauterine Growth Restriction Is Associated with Unique Features of the Reproductive Microbiome. Reprod Sci, 2021. 28(3): p. 828-837.

36. Walther-Antonio, M.R., et al., Potential contribution of the uterine microbiome in the development of endometrial cancer. Genome Med, 2016. 8(1): p. 122.

\section{Figures}



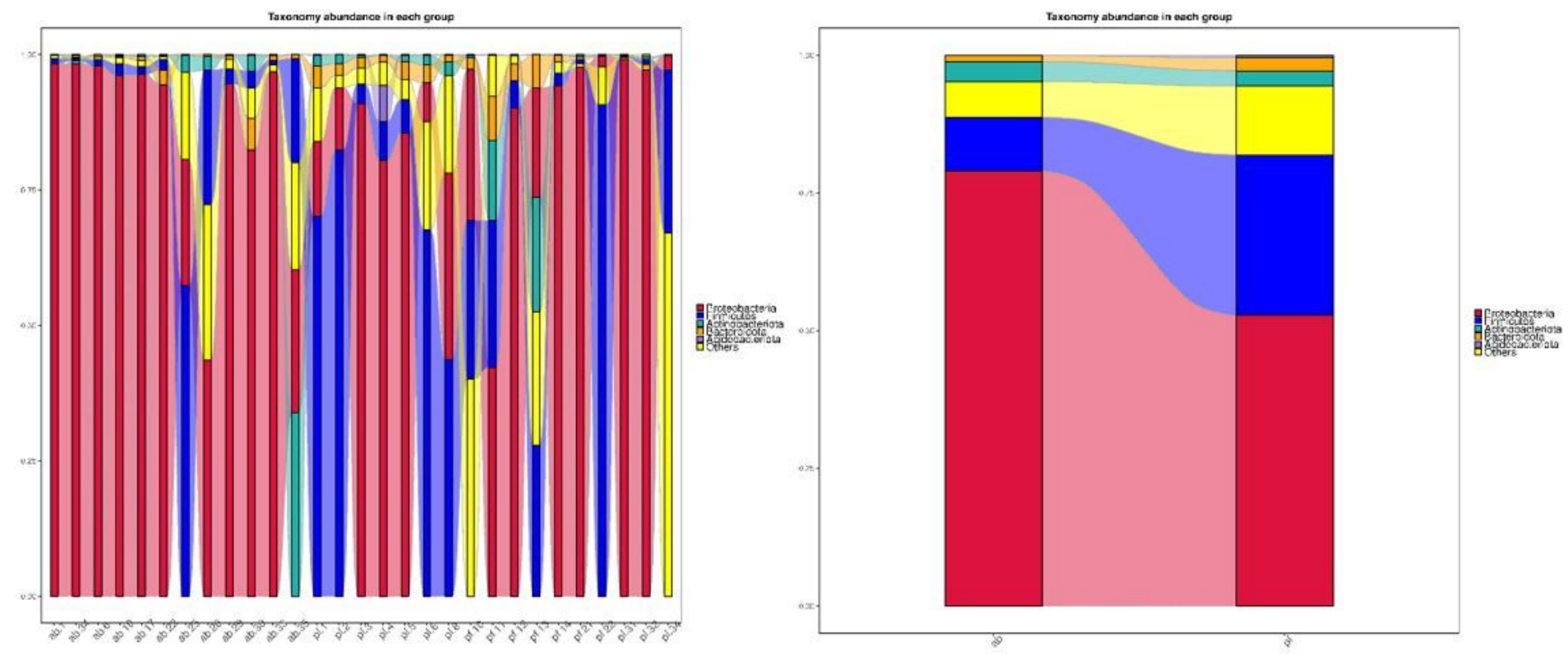

\section{Figure 1}

The abscissa of Sangji diagram is the sample name, the ordinate is the relative abundance of the top 5 species selected, and others represents the sum of the relative abundance of all other species except these 5 species. (ab means the normal pregnant and pf means the missed abortion)
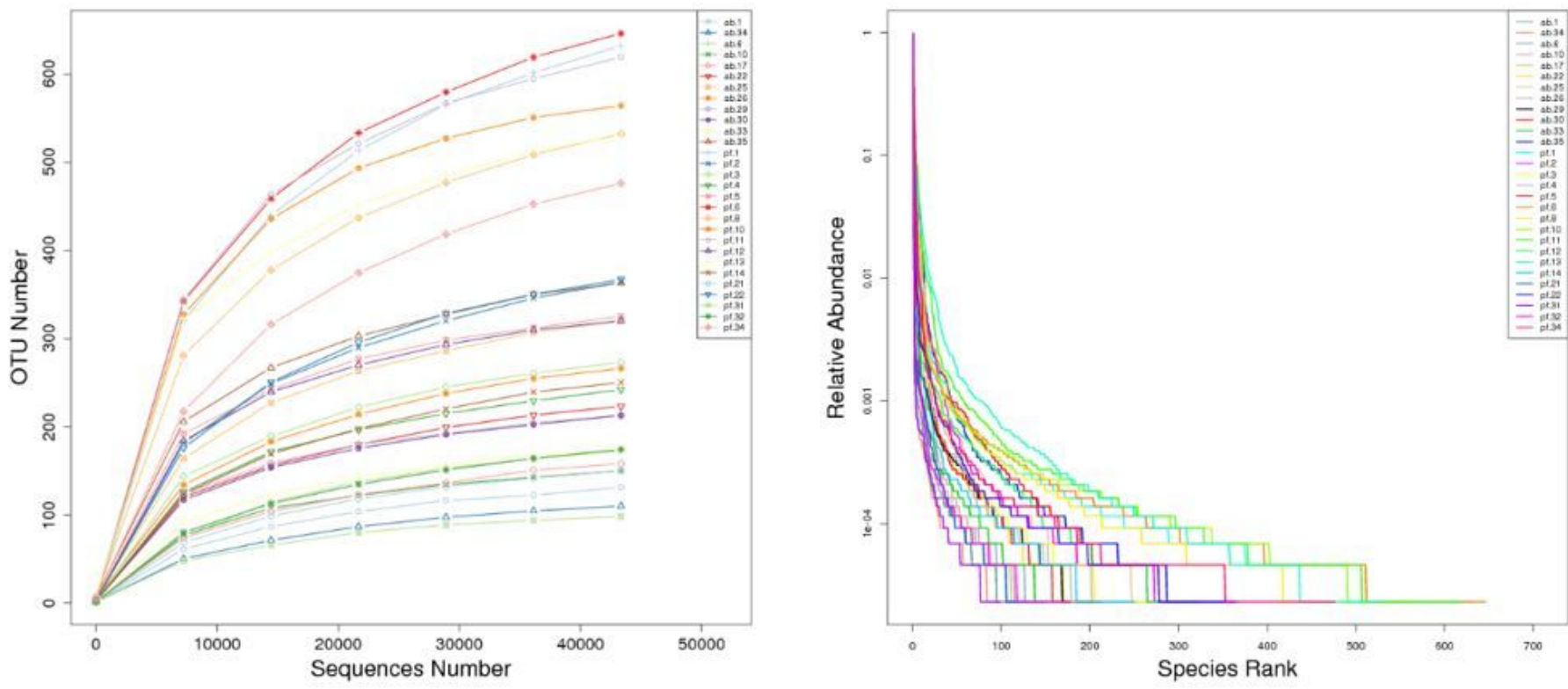

\section{Figure 2}

a) Dilution curve. The abscissa is the number of sequencing strips randomly selected from a sample, and the ordinate is the number of OTU obtained based on the number of sequencing strips. Different samples are represented by curves of different colors. b) Rank Abundance, the abscissa is the sequence number ranked by OTUs Abundance, the ordinate is the relative Abundance of the corresponding OTUs, and different samples are represented by broken lines of different colors. 


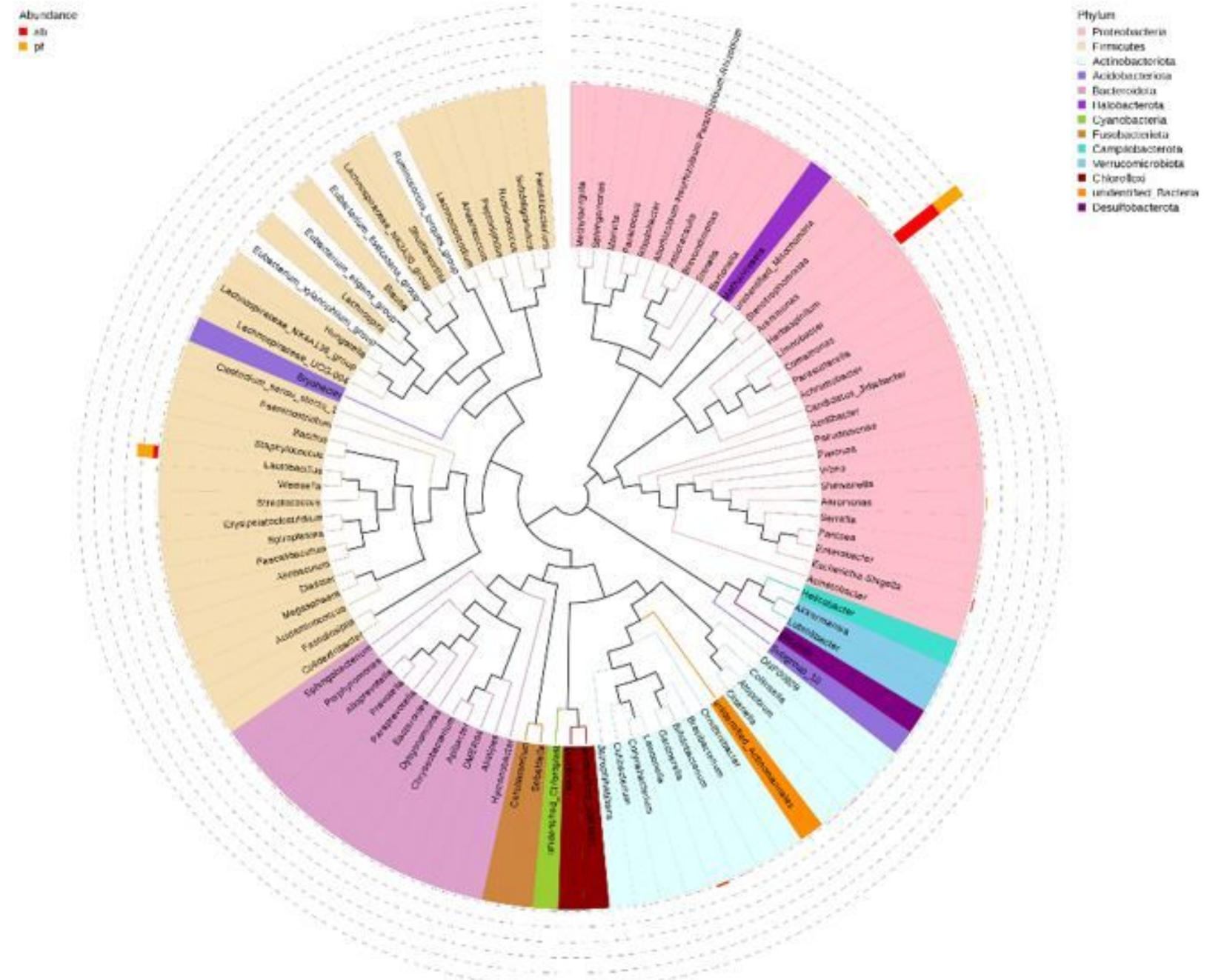

Figure 3

Phylogenetic relationships of species at genus level, the phylogenetic tree is constructed from the representative sequences of species at the level of genus. The colors of branches and fan shapes represent their corresponding phyla. The stacked bar diagrams outside the fan rings represent the abundance distribution information of this genus in different samples. 

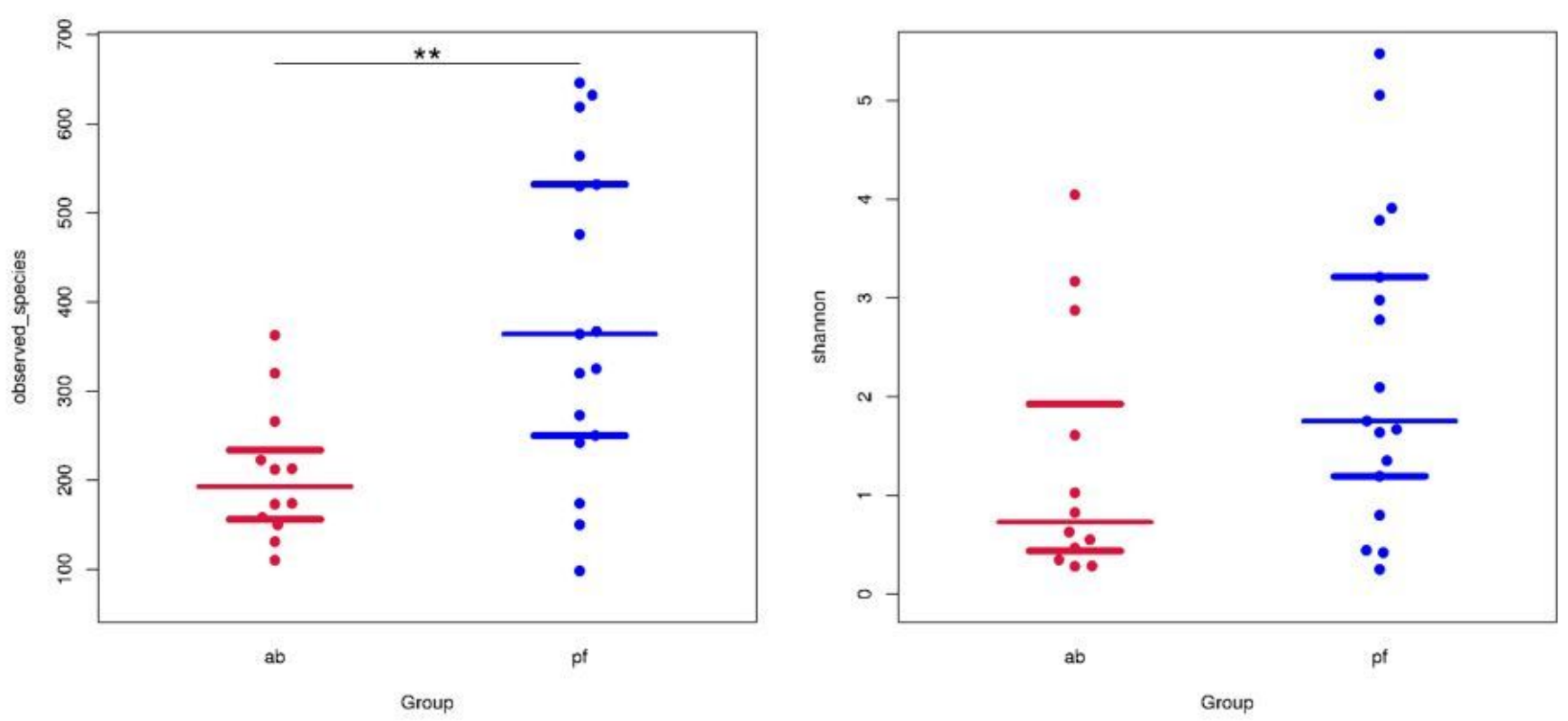

Figure 4

Beeswarm. a) The abscissa is the group name, and the ordinate is the value of the observed species index. b) The abscissa is the group name, and the ordinate is the value of Shannon index value.
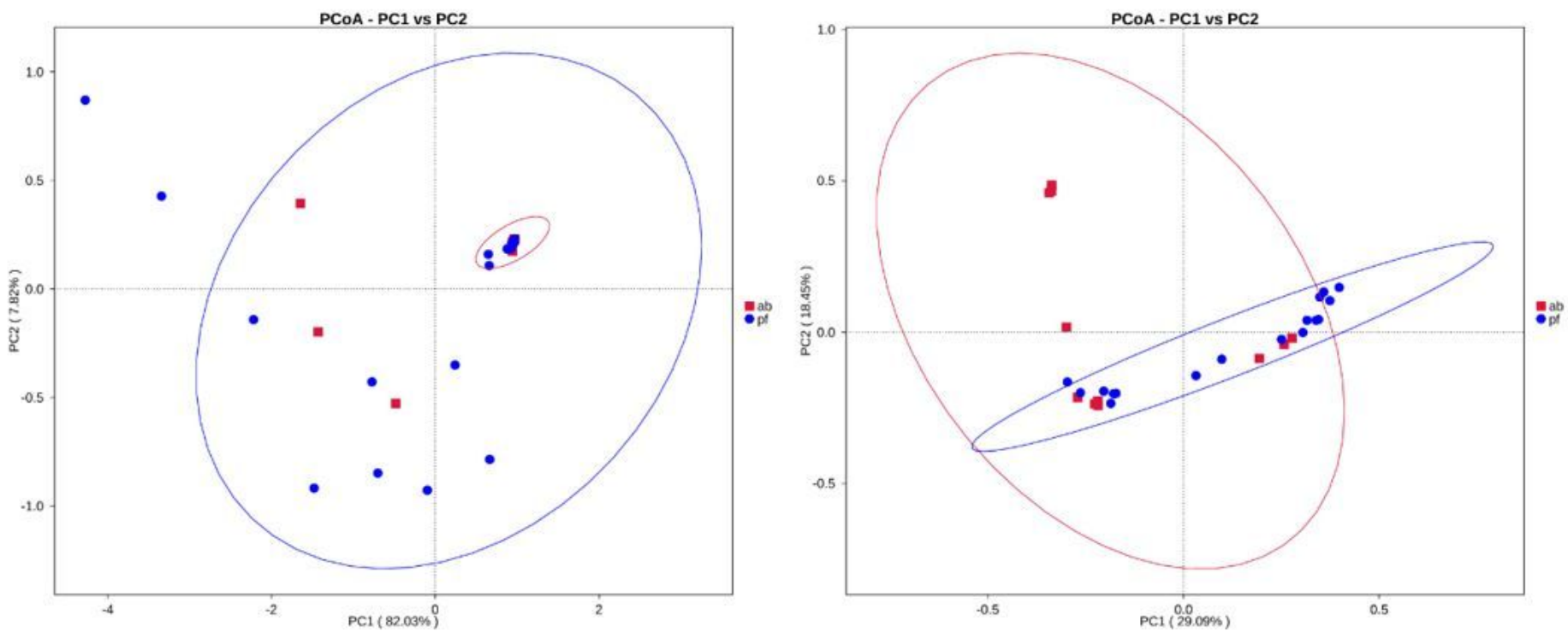

Figure 5

The abscissa and ordinate are the first principal component and the second principal component respectively, and the percentage represents the interpretation rate of principal component to sample difference; Each point in the figure represents a sample, and the samples of the same group are represented by the same color. 


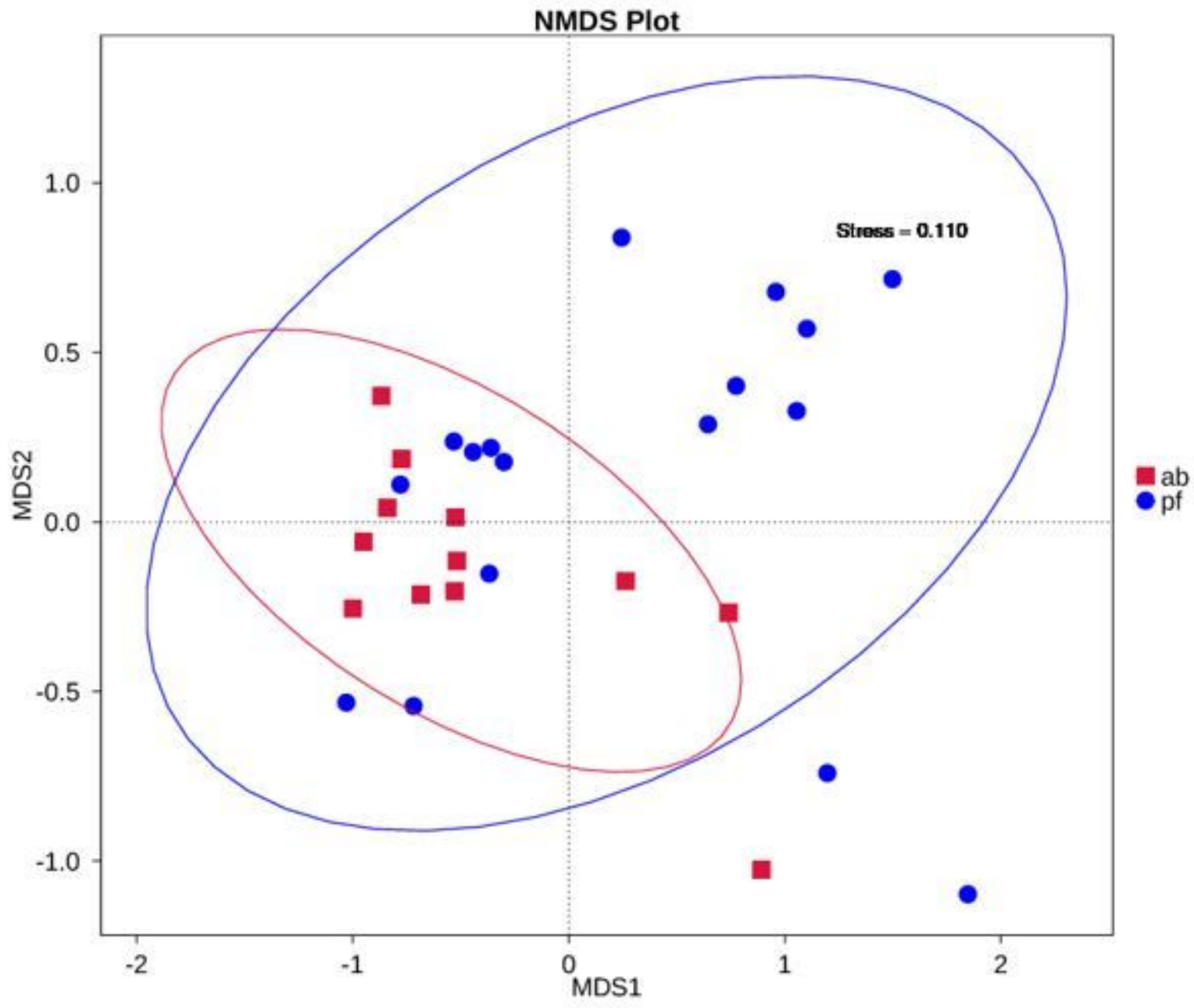

Figure 6

Each point in the diagram represents a sample, the distance between the points indicates the degree of difference, and samples in the same group are represented by the same color. The smaller the Stress $(<0.2)$, the more accurately NMDS can reflect the difference between samples.
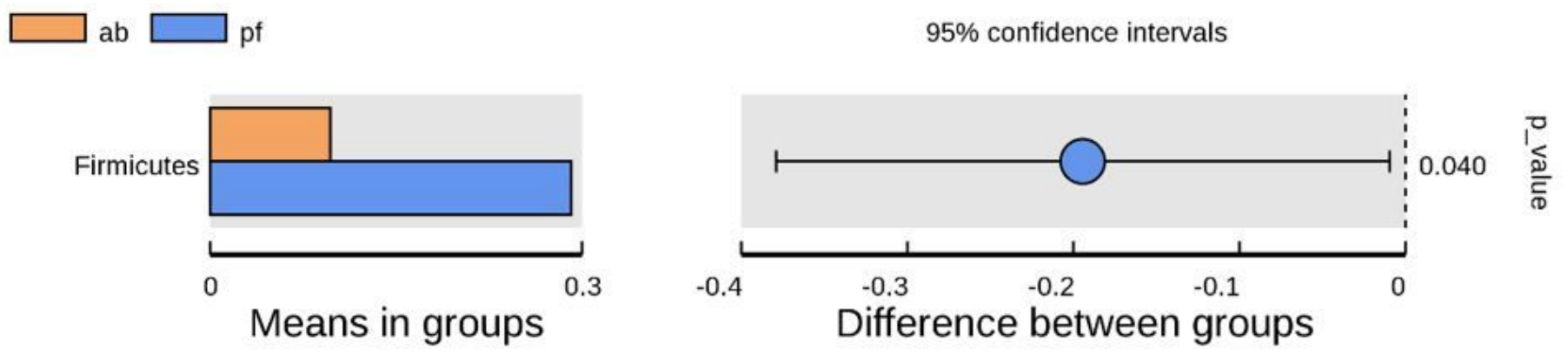

Figure 7

T_ test analysis species difference between test groups. the left figure shows the species abundance of the difference between groups, and each bar in the figure represents the mean value of species with 
significant difference in abundance between groups. the right figure shows the confidence of inter group differences.

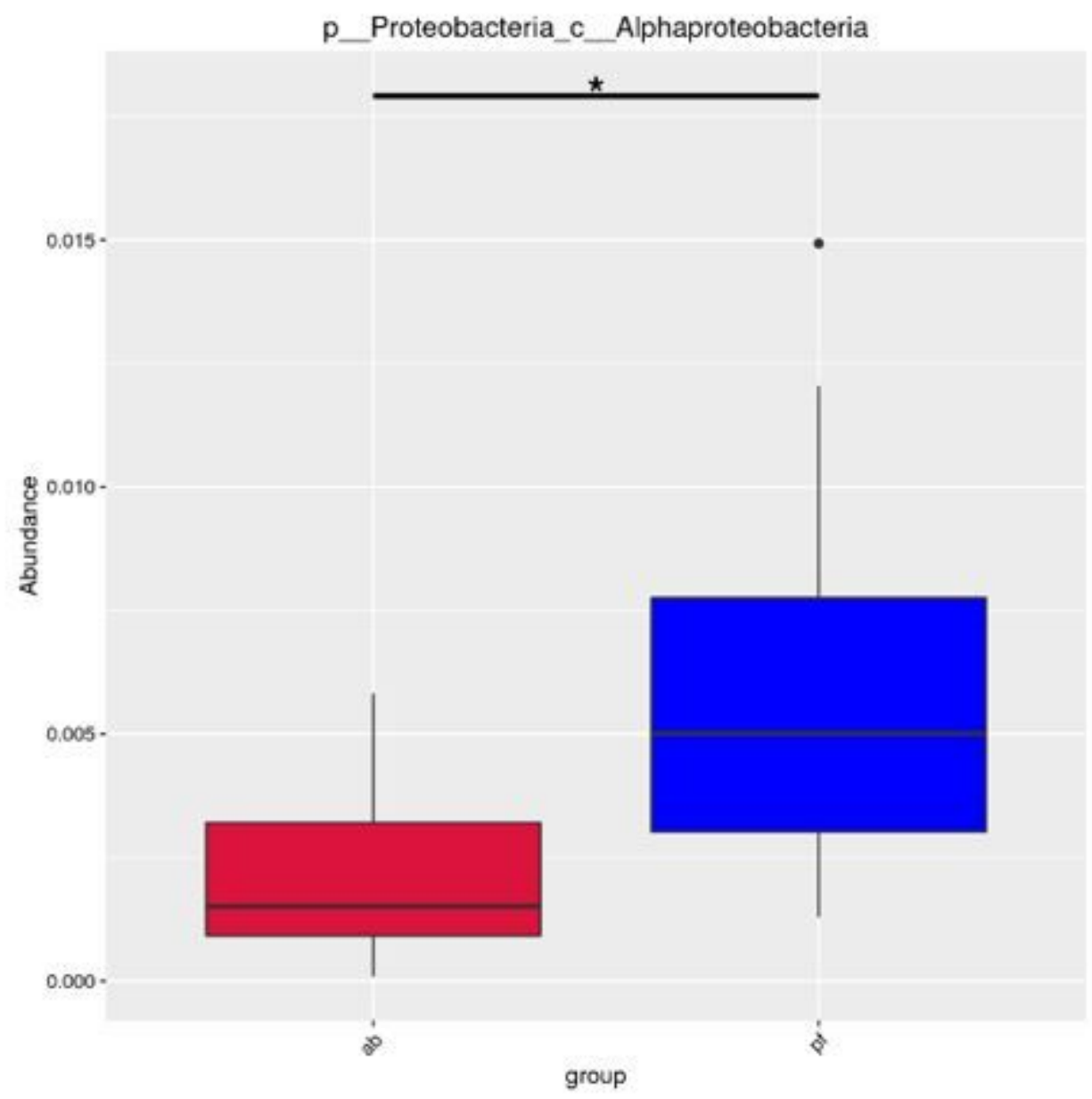

\section{Figure 8}

the horizontal axis is sample grouping; The vertical is the relative abundance of corresponding species.
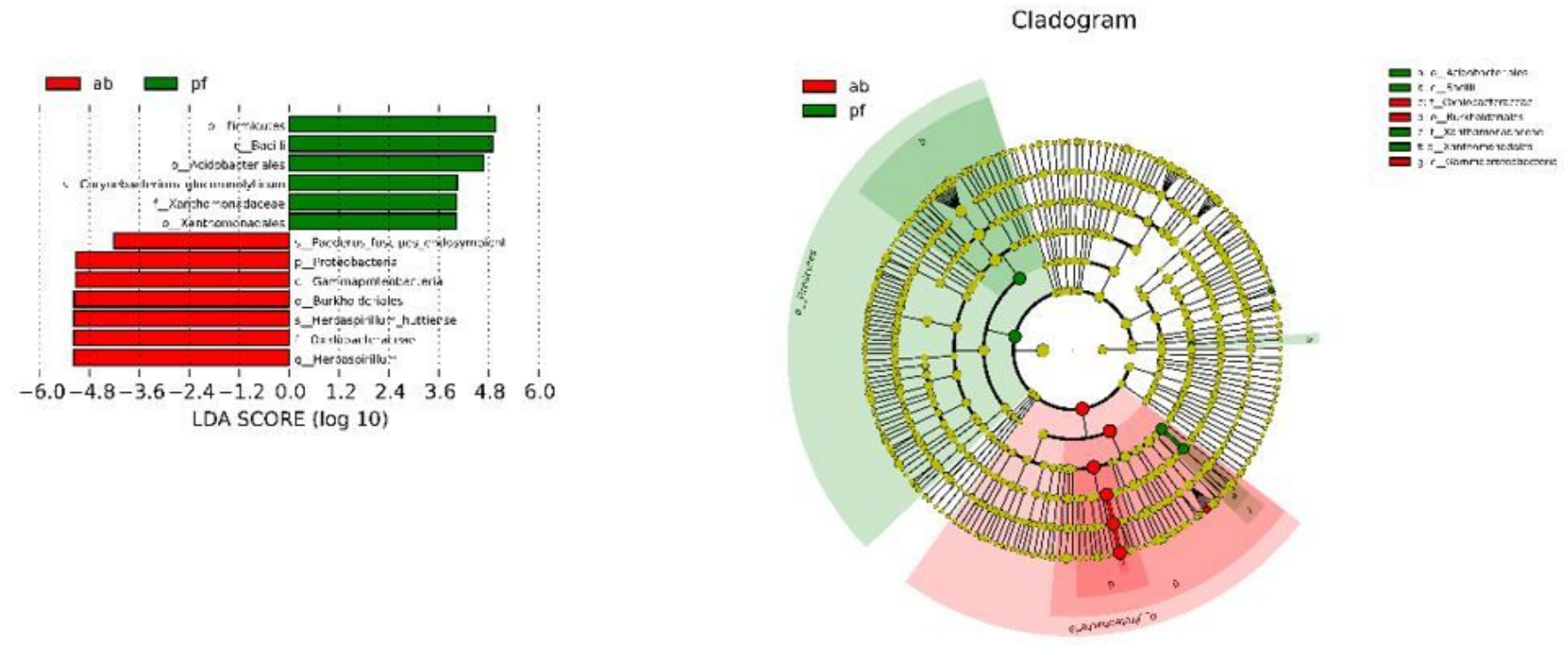


\section{Figure 9}

The histogram of LDA value distribution showed the species with LDA score greater than 4 (biomarker); The cladogram shows the abundance (circle size) from phylum to genus / species (circle from inside to outside) and the importance in a group (the same coloring as the group indicates that it is more important in the group).

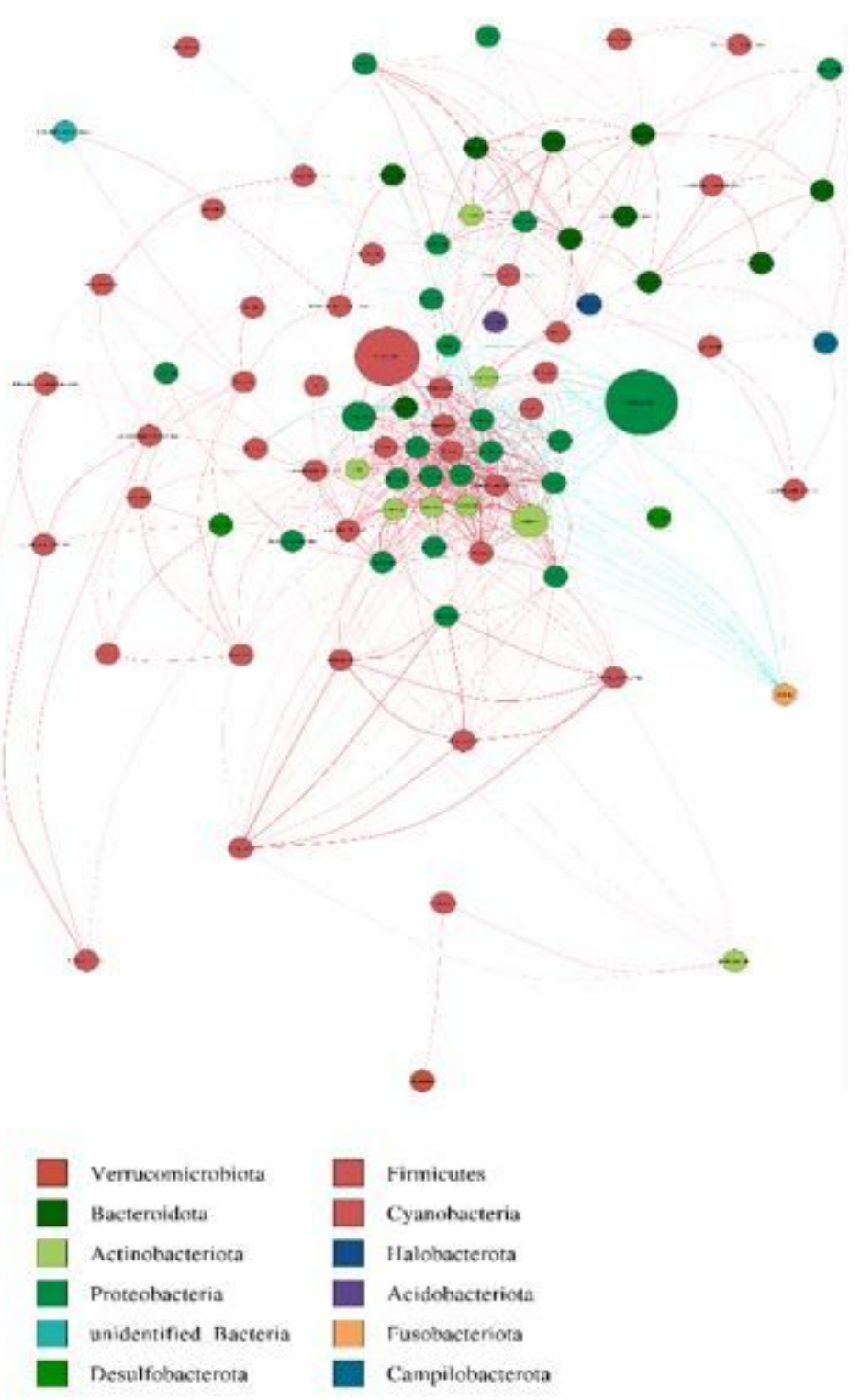

\section{Figure 10}

Note: different nodes represent different genera, node size represents the average relative abundance of the genus, node color of the same door is the same (as shown in the figure), the thickness of the connecting line between nodes is positively correlated with the absolute value of the correlation coefficient of species interaction, and the color of the connecting line is positively correlated with the correlation (red positive correlation, blue negative correlation). 